\title{
Protocol
}

\section{Isolation and Laboratory Domestication of Natural Yeast Strains}

\author{
Gianni Liti, ${ }^{1,4}$ Jonas Warringer, ${ }^{2,3}$ and Anders Blomberg ${ }^{2}$ \\ ${ }^{1}$ IRCAN, CNRS UMR 6267, INSERM U998, University of Nice, 06107 Nice, France; ${ }^{2}$ Department of Chemistry \\ and Molecular Biology, University of Gothenburg, 40530 Gothenburg, Sweden; ${ }^{3}$ Centre for Integrative Genetics \\ (CIGENE), Department of Animal and Aquacultural Sciences, Norwegian University of Life Sciences (UMB), \\ 1432 As, Norway
}

The process from yeast isolation to their use in laboratory experiments is lengthy. Historically, Saccharomyces strains were easily obtained by sampling alcoholic fermentation processes or other substrates associated with human activity in which Saccharomyces was heavily enriched. In contrast, wild Saccharomyces yeasts are found in complex microbial communities and small population sizes, making isolation challenging. We have overcome this problem by enriching yeast on media favoring the growth of Saccharomyces over other microorganisms. The isolation process is usually followed by molecular characterization that allows the strain identification. Finally, yeast isolated from domestic or wild environments need to be genetically manipulated before they can be used in laboratory experiments.

Reagents

It is essential that you consult the appropriate Material Safety Data Sheets and your institution's Environmental Health and Safety Office for proper handling of equipment and hazardous material used in this protocol.

RECIPES: Please see the end of this protocol for recipes indicated by $<R>$. Additional recipes can be found online at http://cshprotocols.cshlp.org/site/recipes.

Equipment

Dry incubator (temperature-controlled)

Petri dishes

Sample collection materials (e.g., gloves, sterile cotton balls, scalpels, tweezers, and smart phone)

Shaking incubator (for 30-50 mL tubes; temperature-controlled)

Tubes (sterile; $30-50 \mathrm{~mL}$ )

\footnotetext{
${ }^{4}$ Correspondence: gianni.liti@unice.fr

(C) 2017 Cold Spring Harbor Laboratory Press

Cite this protocol as Cold Spring Harb Protoc; doi:10.1101/pdb.prot089052
} 
Sampling

1. Collect substrates from wild or human-associated environments. Gather a few cubic centimeters of solid substrates (e.g., tree bark, fruit, soil, mushrooms, and insects) or a few milliliters of liquid substrates (e.g., fermenting must, tree sap, and nectar). Alternatively, rub a sterile cotton ball on a surface (e.g., a fermentation vessel).

Avoid contamination by using gloves and sterilized tools.

2. Meticulously record a description of the sampled item (e.g., take a photograph, note which part of the plant was sampled, record the date, and use a smart phone to precisely record the global positioning system coordinates of the collection site).

In the future, we hope that such documentation will become compulsory information to report when new strains are isolated.

\section{Enrichment and Isolation}

3. Incubate each sample in the Saccharomyces sensu stricto enrichment medium at $25^{\circ} \mathrm{C}$. Break solid samples into smaller pieces before placing them in the enrichment medium.

See Troubleshooting.

4. After $7 \mathrm{~d}$, look for signs of fermentation (i.e., an increase in turbidity and $\mathrm{CO}_{2}$ production). Discard cultures that lack these characteristics.

5. Dilute the actively fermenting cultures, and spread appropriate volumes $(\sim 100 \mu \mathrm{L})$ on YPD agar plates (or plates with a composition resembling the enrichment medium). Incubate for $2-5 \mathrm{~d}$ at $25^{\circ} \mathrm{C}$.

6. Check the color and morphology (size and shape) of colonies by microscopy, and discard any obvious nonyeast microbes (e.g., bacteria). Retain those resembling Saccharomyces colonies for further analysis.

7. Incubate colonies that resemble yeast in potassium acetate medium at $23^{\circ} \mathrm{C}$.

Potassium acetate medium is a respiratory medium lacking nitrogen that induces Saccharomyces meiosis.

8. After 3-7 d of sporulation, look for the presence of ascus structures containing four spores, which is a distinctive trait of Saccharomyces cerevisiae-related yeasts. Saccharomyces sensu stricto strains can be stored indefinitely in the $-80^{\circ} \mathrm{C}$ laboratory freezer in $25 \%$ sterile glycerol solution.

Species Identification

9. Use a molecular method to identify the yeast species.

The choice of the method depends on the equipment available and personal expertise. A combination of screening procedures may be used. We favor sequencing a small fragment of rDNA (e.g., ITS1 and ITS2; James et al. 1996) and pulsed-field gel electrophoresis karyotyping for an unequivocal identification of different Saccharomyces sensu stricto species (Liti et al. 2005). The karyotype of Saccharomyces sensu stricto yeast species is distinct from that of other yeast species with a larger number of smaller chromosomes. Karyotyping also provides information about chromosomal rearrangements and potential strain hybridization (Liti et al. 2005). Restriction analysis of rDNA and mtDNA can give an indication of the species as can a number of recently developed species-specific primers (Muir et al. 2011). An alternative method is the use of tester strains and genetic crosses for the biological identification of the species (Naumov et al. 2010). This test relies on measuring gamete viability in crosses with species representatives.

Preparing Strains for Laboratory Work

10. Once the species has been identified, perform further molecular characterization, phenotyping, and/or genetic manipulation in preparation for future work.

The genetic manipulation is dictated by the nature of the downstream analysis. The majority of wild strains are diploid homothallic, harbor varying levels of heterozygosity, and are mostly, but not always, able to grow 
G. Liti et al.

in minimal medium. The removal of heterozygosity is generally required. This is performed by isolating monosporic derivatives, which otherwise will segregate in downstream steps. Deletion of the HO gene using a dominant resistance marker amplified from the drug-resistance plasmids pFA6 (kanMX4), pAG25 (natMX4), or pAG32 (hphMX4) produces stable haploid strains (Goldstein and McCusker 1999).

The availability of inexpensive restriction site-associated DNA (RAD) sequencing approaches allows a deep analysis of genome phylogeny and structure (Cromie et al. 2013). Molecular barcodes can be added in the polymerase chain reaction construct to uniquely label strains (Cubillos et al. 2009). Additional auxotrophic markers can be introduced by using the marker-less plasmids strategy (Louvel et al. 2014). Marker-less plasmids include pAD1 (LEU2), pAD2 (LYS2), and pAD4 (MET15); for further details, see Brachmann et al. (1998). A standard transformation protocol works well in the majority of strains, although efficiency varies greatly depending on genetic background and conditions may need adjustment (see Troubleshooting).

\section{TROUBLESHOOTING}

Problem (Step 3): Growth conditions for recovery of the new strain are unknown.

Solution: Incubation at $30^{\circ} \mathrm{C}$ tends to favor S. cerevisiae strains and closely related species (e.g., S. paradoxus and S. mikatae). A lower incubation temperature (e.g., $23^{\circ} \mathrm{C}-25^{\circ} \mathrm{C}$ ) will recover a broader range of Saccharomyces species (Sampaio and Gonçalves 2008). The Saccharomyces sensu stricto enrichment medium preferentially selects Saccharomyces over other microorganisms (Naumov et al. 1997; Wang et al. 2012). It may be necessary to make adjustments to the concentration of components of this enrichment medium because different species display quantitative variation in tolerance to these reagents (e.g., to ethanol). A possible solution is to split the sample into aliquots and test different enrichment conditions on these samples, thereby maximizing chances of recovering multiple species and strains.

Problem (Step 10): Some isolates perform very badly in laboratory protocols for sporulation, spore viability, and transformation efficiency.

Solution: The availability of multiple isolates from the same area or substrate might help with the selection of the right strain to work with. There is a considerable variation in these phenotypes, even in closely related strains (e.g., because of the presence of rare loss-of-function alleles; Bergström et al. [2014]). Karyotype analysis can help to identify hybrid Saccharomyces strains, which are interesting from an ecological and evolutionary perspective, but are not amenable to genetic manipulation (e.g., as a result of poor spore viability). Protocols may need to be strain optimized. For example, strains respond differently to sporulation conditions (e.g., liquid or solid, potassium acetate medium or minimal potassium acetate medium). Similarly, several steps of the lithium acetate transformation protocol can be adjusted to optimize the transformation process. We suggest testing multiple volumes for re-inoculation after the overnight cultivation. Another critical parameter is the length of the $42^{\circ} \mathrm{C}$ heat shock. We observed increased transformation efficiency in multiple isolates by extending the incubation time to $30-45 \mathrm{~min}$.

Increasing interest in the ecology and evolution in natural habitat of the budding yeast $S$. cerevisiae has prompted field surveys aimed at isolating natural strains (Hittinger 2013; Boynton and Greig 2014). A landmark study that focused on several areas of China allowed isolation of hundreds of S. cerevisiae isolates from many environments not previously probed (Wang et al. 2012). These studies documented the presence of $S$. cerevisiae in wild settings but also indicated that the success of isolation is low, perhaps caused by low population size. Isolation based on enrichment media allows specific species to be recovered. Minor differences in environmental factors (e.g., temperature) can favor one species over another (Sampaio and Gonçalves 2008). Future metagenomic studies have the potential to give a better understanding of the microbial communities before selection by enrichment media. 
Yeast geneticists are also increasingly exploiting natural variation as a tool to understand the relationship between genotype and phenotype (Nieduszynski and Liti 2011; Fay 2013). For example, see Protocol: Mapping Quantitative Trait Loci in Yeast (Liti et al. 2015). The "raw material" for these experiments is naturally diverged yeast strains, such as those isolated using the procedures described above.

\section{RECIPES}

COM Drop-Out Powder

\begin{tabular}{lr} 
Reagent & Quantity \\
\hline Adenine & $800 \mathrm{mg}$ \\
Arginine & $800 \mathrm{mg}$ \\
Aspartic acid & $4000 \mathrm{mg}$ \\
Histidine & $800 \mathrm{mg}$ \\
Leucine & $800 \mathrm{mg}$ \\
Lysine & $1200 \mathrm{mg}$ \\
Methionine & $800 \mathrm{mg}$ \\
Phenylalanine & $2000 \mathrm{mg}$ \\
Threonine & $8000 \mathrm{mg}$ \\
Tryptophan & $800 \mathrm{mg}$ \\
Tyrosine & $1200 \mathrm{mg}$ \\
Uracil & $800 \mathrm{mg}$
\end{tabular}

Mix the reagents together, and store the powder at room temperature.

Potassium Acetate Medium (Liquid or Solid)

Reagent Quantity (for 1 L)

Potassium acetate $16 \mathrm{~g}$

Yeast extract $1.76 \mathrm{~g}$

Dextrose $0.4 \mathrm{~g}$

COM drop-out powder $<\mathrm{R}>$

$0.7 \mathrm{~g}$

Agar (for solid medium only)

$20 \mathrm{~g}$

Dissolve the potassium acetate, yeast extract, and dextrose in $800 \mathrm{~mL}$ of distilled water while stirring continuously. Adjust the $\mathrm{pH}$ to 7.0 with $1 \mathrm{~m} \mathrm{HCl}$ or $2.5 \mathrm{M} \mathrm{NaOH}$. Add the COM drop-out powder and the agar (if used). Adjust the volume to $1 \mathrm{~L}$. Autoclave at 0.5 bar for $20 \mathrm{~min}$ at $121^{\circ} \mathrm{C}$. Store for several weeks at room temperature.

Saccharomyces Sensu Stricto Enrichment Medium

Reagent

Quantity (for 1 L)

Yeast extract

$3 \mathrm{~g}$

Malt extract

$3 \mathrm{~g}$

Bacto peptone

$5 \mathrm{~g}$

Glucose

$10 \mathrm{~g}$

Ethanol

$80 \mathrm{~mL}$

Chloramphenicol

$200 \mathrm{mg}$

$\mathrm{HCl}(1 \mathrm{M})$

$1 \mathrm{~mL}$

Dissolve the reagents in a final volume of $1 \mathrm{~L}$ of distilled water. Autoclave at 0.5 bar for $20 \mathrm{~min}$ at $121^{\circ} \mathrm{C}$. Store for several weeks at room temperature. 
G. Liti et al.

Yeast Extract-Peptone-Dextrose (YPD) Medium (Liquid or Solid)

Reagent Quantity

$\begin{array}{ll}\text { Yeast extract } & 10 \mathrm{~g}\end{array}$

$\begin{array}{ll}\text { Peptone } & 20 \mathrm{~g}\end{array}$

$\begin{array}{ll}\text { Dextrose } & 20 \mathrm{~g}\end{array}$

Agar (for plates only) $\quad 20 \mathrm{~g}$

Dissolve reagents in $1 \mathrm{~L}$ of distilled water. Autoclave for $20 \mathrm{~min}$ at $121^{\circ} \mathrm{C}$ and $0.5 \mathrm{bar}$.

Store for several weeks at room temperature.

\section{REFERENCES}

Bergström A, Simpson JT, Salinas F, Barré B, Parts L, Zia A, Nguyen Ba AN, Moses AM, Louis EJ, Mustonen V, et al. 2014. A high-definition view of functional genetic variation from natural yeast genomes. Mol Biol Evol 31: 872-888.

Boynton PJ, Greig D. 2014. The ecology and evolution of non-domesticated Saccharomyces species. Yeast 31: 449-462.

Brachmann CB, Davies A, Cost GJ, Caputo E, Li J, Hieter P, Boeke JD. 1998. Designer deletion strains derived from Saccharomyces cerevisiae S288C: A useful set of strains and plasmids for PCR-mediated gene disruption and other applications. Yeast 14: 115-132.

Cromie GA, Hyma KE, Ludlow CL, Garmendia-Torres C, Gilbert TL, May P, Huang AA, Dudley AM, Fay JC. 2013. Genomic sequence diversity and population structure of Saccharomyces cerevisiae assessed by RADseq. G3 (Bethesda) 3: 2163-2171.

Cubillos FA, Louis EJ, Liti G. 2009. Generation of a large set of genetically tractable haploid and diploid Saccharomyces strains. FEMS Yeast Res 9: $1217-1225$.

Fay JC. 2013. The molecular basis of phenotypic variation in yeast. Curr Opin Genet Dev 23: 672-677.

Goldstein AL, McCusker JH. 1999. Three new dominant drug resistance cassettes for gene disruption in Saccharomyces cerevisiae. Yeast 15: 1541-1553.

Hittinger CT. 2013. Saccharomyces diversity and evolution: A budding model genus. Trends Genet 29: 309-317.

James SA, Collins MD, Roberts IN. 1996. Use of an rRNA internal transcribed spacer region to distinguish phylogenetically closely related species of the genera Zygosaccharomyces and Torulaspora. Int J Syst Bacteriol 46: 189-194.
Liti G, Peruffo A, James SA, Roberts IN, Louis EJ. 2005. Inferences of evolutionary relationships from a population survey of LTR-retrotransposons and telomeric-associated sequences in the Saccharomyces sensu stricto complex. Yeast 22: 177-192.

Liti G, Warringer J, Blomberg A. 2015. Mapping quantitative trait loci in yeast. Cold Spring Harb Protoc doi: 10.1101/pdb.prot089060.

Louvel H, Gillet-Markowska A, Liti G, Fischer G. 2014. A set of genetically diverged Saccharomyces cerevisiae strains with markerless deletions of multiple auxotrophic genes. Yeast 31: 91-101.

Muir A, Harrison E, Wheals A. 2011. A multiplex set of species-specific primers for rapid identification of members of the genus Saccharomyces. FEMS Yeast Res 11: 552-563.

Naumov GI, Naumova ES, Sniegowski PD. 1997. Differentiation of European and Far East Asian populations of Saccharomyces paradoxus by allozyme analysis. Int J Syst Bacteriol 47: 341-344.

Naumov GI, Naumova ES, Masneuf-Pomarède I. 2010. Genetic identification of new biological species Saccharomyces arboricolus Wang et Bai. Antonie Van Leeuwenhoek 98: 1-7.

Nieduszynski CA, Liti G. 2011. From sequence to function: Insights from natural variation in budding yeasts. Biochim Biophys Acta 1810: 959966.

Sampaio JP, Gonçalves P. 2008. Natural populations of Saccharomyces kudriavzevii in Portugal are associated with oak bark and are sympatric with S. cerevisiae and S. paradoxus. Appl Environ Microbiol 74: 21442152.

Wang Q-M, Liu W-Q, Liti G, Wang S-A, Bai F-Y. 2012. Surprisingly diverged populations of Saccharomyces cerevisiae in natural environments remote from human activity. Mol Ecol 21: 5404-5417. 


\section{Isolation and Laboratory Domestication of Natural Yeast Strains}

Gianni Liti, Jonas Warringer and Anders Blomberg

Cold Spring Harb Protoc; doi: 10.1101/pdb.prot089052

\begin{tabular}{cc}
$\begin{array}{r}\text { Email Alerting } \\
\text { Service }\end{array}$ & Receive free email alerts when new articles cite this article - click here. \\
\hline $\begin{array}{c}\text { Subject } \\
\text { Categories }\end{array}$ & $\begin{array}{c}\text { Browse articles on similar topics from Cold Spring Harbor Protocols. } \\
\text { Laboratory Organisms, general (924 articles) } \\
\text { Yeast (288 articles) }\end{array}$ \\
\hline
\end{tabular}

